\title{
Ruptura y mutación. \\ Sobre el cambio del mundo de la vida en Alemania del Este y del Oeste
}

\section{Rainer Zoll}

Universidad de Bremen. Departamento de Sociología. Alemania

\section{Resumen}

El cambio social en Alemania del Este ha provocado una fractura en el mundo de la vida de esta sociedad que se manifiesta en diversas patologías sociales (pérdida de confianza, crisis de identidad, etc.). (Redacción)

Palabras clave: mundo de la vida, cambio sociocultural, crisis.

\section{Abstract. Break and Mutation. Changes in West and East Germany world life}

Social change in East Germany has produced a break of the life-world, which can be seen in the form of several social patologies (loss of trust, identity crisis, etc.). (Redaction)

Key words: life-world, socio-cultural change, crisis.

\section{Sumario}

El ejemplo de la Alemania del Este

Ruptura y cambio

Bibliografía

Tesis: La ruptura social en Alemania del Este y el cambio radical del mundo de la vida ocultan - al menos patcialmente- la mutación del mundo de la vida en Alemania del Oeste. En Alemania no solamente vivimos en una situación donde las seguridades del mundo de la vida que rienen una significación existencial para los seres humanos son amenazadas sino también muchas veces ya destruidas.

* Transcripción de la Conferencia de R. Zoll en el Departamento de Sociología de la Universirae Auronoma de Barcelona, en marzo de 1993. 
Pero antes de entrar en los detalles de esta interpretación, me parece necesario comentar el concepto de mundo de la vida (Lebenswelt) y de precisar mi versión de la noción. Esta necesidad resulta del hecho que la expresión "mundo de la vida" parece muy fácil de comprender pero en realidad significa un nexo bastante complejo. Además existen algunas versiones diferentes del concepto. Quisiera notar en seguida que hay una valoración positiva del concepto porque se relaciona con vida, y hoy - que la vida está tan amenazada - todo lo que se refiere a la vida tiene una valoración positiva. A pesar de esto, el mundo de la vida es un concepto analíico que no contiene una valoración a priori.

En lo que sigue me refiero al concepto de mundo de la vida de Alfred Schüitz y Thomas Luckmann y no a las versiones de Habermas o de Husserl. Para ellos el mundo de la vida es un concepto a metanivel que significa más bien las estructuras que están detrás del mundo de la vida concreto y cotidiano en el sentido de Schütz/Luckmann --estructuras que determinan el mundo de la vida- - Pero en efecto acepto la crítica de Habermas dirigida a Schütz/Luckmann acerca de que el concepto de mundo de la vida debería ser integrado en una teoría de la sociedad. Pienso también que la tentativa de Habermas de satisfacer esta exigencia con la diferenciación entre sistema y mundo de la vida tiene sentido. Para Habermas el mundo de la vida es la esfera de la vida privada y la esfera pública, mientras que el sistema contiene la economía y el estado designa la estructura institucional de la sociedad-. Pero opino que el empleo de la palabra "sistema" es desafortunado para designar la esfera de la sociedad que es la estructura del poder institucionalizado de la política y de la economía, porque la deducción de la noción de sistema en este sentido de la teoría de los sistemas no me parece imperativa ni concluyente, al contrario. La nueva teoría de los sistemas aplica la noción de sistema a todas las unidades sociales, orgánicas y otras; un sistema puede ser en este sentido un hombre, un estado, una sociedad, un animal, una planta, pero también un biotopo, un peer-group, una secta de jóvenes, un partido político, etc. En base a la teoría de los sistemas no es convincente acentuar más que por otros sistemas el carácter de sistema de la estructuta de poder institucionalizado en la política y la economía designándola preferentemente como sistema. Cierto que es un sistema pero también el mundo de la vida puede ser interpretado como un sistema con sus propias leyes de reproducción. Particularmente desafortunada me parece sin embargo la afinidad del concepto habermasiano de sistema con la noción de sistema de los nacionalsocialistas, de los nazis, que han designado con esta palabra a la sociedad burguesa y su estructura institucional.

Con la descripción de las "estructuras del mundo de la vida" Schütz y Luckmann quieren evidenciar una dimensión de la vida humana hasea entonces apenas considerada en las ciencias sociales. Por cierto, es casi banal determinar el mundo de la vida como lo cotidiano, como lugar de la comunicación y de la acción humana, la cual está limirada por otros seres humanos, por la naturaleza y por la sociedad. El mundo de la vida no es mi mundo privado, es intersubjetivo, lo convivido con otras personas. Puedo experimentarlo sensualmente; eilo comprende la naturaleza, el mundo social y el mundo cultural. 
Pero ante todo Schütz y Luckmann hacen resaltar que el mundo de la vida nos parece como simplemente dado por supuesto, que es evidente e indudable para nosotros. Esta propiedad del mundo de la vida, su carácter indudable, quedaba oculto durante largo tiempo precisamente porque ello es asi, porque es indudable, simplemente dado y evidente.

La pregunta es entonces cómo algo llega a formar parte del mundo de la vida, cómo algo obtiene estas propiedades: el mundo de la vida debe ser comprendido, es decir, debe ser interpretado por ser apropiado, por llegar a ser mundo de la vida. Ello debe ser familiar en una cierta medida que no llega a ser sin un proceso de familiarización. El proceso de interpretación y de familiarización, sin embargo, no se basa solamente en las propias experiencias sino también en experiencias de otros. No adquirimos los esquemas habituales de interpretación y de referencia solamente por experiencias que vivimos e interpretamos nosotros mismos, sino también por experiencias ajenas que asumimos de personas familiares. El proceso de interpretación — por regla general no reflexivo- que efectuamos continuamente, da como resultado un significado, un sentido - sentido que los acontecimientos tienen para nosotros, que la vida tiene para nosotros.

Nuestro repertorio, nuestro stock de saberes cotidianos consiste en capas de sentido, de significación que son sedimentos de interpretaciones pasadas, interpretaciones habitualizadas. El individuo nada como un pez en el agua de las "significaciones implícitas" - como to ha dicho Mary Douglas - y esto significa que el individuo tiene confianza en estas significaciones, en su sentido, en el mundo de la vida.

Los elementos singulares del saber cotidiano no son necesariamente siempre indudables, sino que pueden llegar a ser problemáticos por desplazamientos del horizonte. En este caso el proceso de interpretación, que en principio es infnito, pero en realidad es interrumpido, en algún momento, debe recomenzar de nuevo. Cada vez que una parte del horizonte se aproxima por aplazamiento, o que una experiencia nueva no entra en los esquemas de interpretación existentes hay irritaciones que hacen necesario idealmente un proceso hermenéutico nuevo. Idealmente porque siempre es posible como alternativa negativa el retiro a lo conocido o una asimilación reduccionista de los acontecimientos. Como dicen Schütz y Luckmann, "cada interpretación en el mundo de la vida es una interpretación dentro del marco de lo ya conocido, ya interpretado, dentro del marco de una realidad que, en principio y por sus características, es familiar. Es la confianza de que el mundo como lo conozco continúa siendo como es.n (Schütz/Luckmann, 1979, p. 29). Sobre esto se basa la «idealización" (Husserl) del "continuar como siempre".

La confianza es una condición sine qua non de nuestra vida. Es una confranza en contextos probados.-probados en muchas repeticiones-, contextos que consisten en seres humanos, en instituciones sociales, en asociaciones colectivas, etc. Sabemos por el psicoanálisis que la confianza tiene una dimensión básica que se llama "confianza primordial" y es adquirida en la díada madre/hijo en la más tierna infancia. Sobre esta confranza primordial se basan 
distintos niveles y también diferentes grados de confianza. Cuando la confianza es cuestionada, cuando se pierde, las consecuencias pueden ser muy diferentes, por ejemplo: dudas acerca de una institución, ruptura de una relación personal o hasta una crisis profunda de la identidad.

Los cambios en el mundo de la vida tienen siempre como consecuencia cambios en estas propiedades: algo hasta ahora indudable llega a ser dudoso; algo dado por supuesto hasta ahora es puesto en cuestión; eso que se entiende por sí mismo no es más evidente. Algo familiar es sustituido por algo ajeno, nuevo. En la medida que el cambio es más o menos grande, es necesario un nuevo proceso de interpretación o los individuos afectados pierden la seguridad proveniente del mundo de la vida.

\section{El ejemplo de la Alemania del Este}

También bajo este aspecto la derrota del pretendido socialismo real tenía consecuencias dramáticas para muchas personas. No sólo la miseria económica causada por el paro, la inflación, pensiones módicas, etc., estremecen a la gente, sino también la ruptura del mundo de la vida que provoca en muchos una crisis de identidad incisiva. Intento describir esta ruptura del mundo de la vida en Alemania del Este en algunos aspectos. La ruptura es evidente porque todo lo que fue indudable, todo a lo que la generación más vieja se había acostumbrado en cuarenta años, es decir, todo lo que llegaba a ser mundo de la vida es puesto en tela de juicio, ahora es cuestionable. Esto sc manifiesta particularmente en una de las áreas más importantes del mundo de la vida, en las relaciones sociales.

En la RDA las relaciones sociales fueron mediadas a través de la primacía de las empresas, de las fábricas. Se hizo mucho para reforzar la cohesión de los colectivos de trabajo, de las brigadas. Las empresas disponían de recursos económicos para efectuar este trabajo de relaciones. Fue un medio de control social, de embrigadamiento. La cara visible de esto fueron los cuidados paternalistas que ayudaban a los individuos a encontrarse, pero al mismo tiempo este sistema permitía a la Stasi, servicio estatal de seguridad, infiltrarse en todas partes. Esta fue la cara oculta que hoy sale a la luz del día.

Unos ejemplos de este "trabajo de telaciones": las empresas financiaban círculos de expresión artística y de otras actividades de entretenimiento. Organizaban también una competición pretendidamente socialista entre los colectivos de trabajo. Eltos recibían puntos para actividades sociales, culturales y políticas, como entradas a conciertos, teatros, museos, etc.; si habían reunido bastantes puntos recibían el titulo "colectivo de trabajo socialista" y un pequeño fondo con que podían financiar una fiesta o algo similar. Las empresas organizaban muchas actividades culturales y sociales - un visitante del oeste que quería presenciar un espectáculo o concierto que en principio estaba lleno, tenía siempre una oportunidad de entrar si esperaba hasta el inicio para ver si los colectivos de trabajo habían de verdad ocupado todos los asientos reservados para ellos--. Las empresas decidían sobre la concesión de huer- 
tos familiares y servían de mediador para obtener un piso, es decir, era casi imposible alquilar un piso sin la intervención de un servicio de la empresa que estaba en relación con las cooperativas de viviendas obreras, los AWG. Y realmente hubo condiciones para obtener más o menos fácilmente la entrada en los AWG. Por ejemplo, personas que se casaban para disfrutar de un piso más grande.

En suma, todo esto conducía a una situación en que las relaciones sociales estaban en su mayor parte mediadas directamente o indirectamente por las empresas. Realmente algunas de estas relaciones eran amistades verdaderas, otras, sin embargo, muy superficiales y muchas de la naturaleza que pueden tener cuando son tan evidentes las condiciones paternalistas y de control social. A pesar de todo fueron muy importantes para la gente que de esta manera se incorporaba a un nexo de redes sociales que daba seguridad y que permitía soportar más fácilmente las desventajas materiales de la economí Ilamada socialista y la falta de libertad, la reglamentación política y social. La nivelación casi completa de la sociedad conducía en el mundo de la vida a una incapacidad considerable de manejar diferencias.

Hoy este nexo social mediado por las empresas es radicalmente perturbado, bien porque èstas han cerrado, bien porque han debido declararse en quiebra, bien porque las pocas empresas nuevas que han sustituido a las viejas son ahora capitalistas que no tienen fondos para política social y todavía menos para política cultural. Ciertamente, en las sociedades europeas occidentales y norteamericanas de vez en cuando hay también empresas capitalistas que poner a disposición de sus empleados, en una determinada medida, la mayoría de las veces bastante baja, un fondo para actividades culturales y sociales, pero con la fundación nueva o la importación de empresas en los países del este se crean en general condiciones análogas al capitalismo primitivo en el que tales fondos fueron consideradas bobadas inútiles.

Las consecuencias del derrumbamiento de la mayoría de las empresas por lo que se refiere a las relaciones sociaies son dramáticas. En muchos casos las relaciones son simplemente destruidas porque eran superficiales o porque las personas han sido separadas. Unos encuentran un puesto de trabajo en Alemania del Oeste, otros en un lugar lejos de su país y otros están parados o retirados o reciben una pensión anticipada. Desde luego hay también amistades que han pervivido al derrumbamiento de la RDA, pero también en estos casos parece que existe como un vaivén, es decir, primero una parálisis debida al derrumbamiento y después de un cierto tiempo, una reanudación. La parálisis fue al mismo tiempo una caída en un agujero psíquico profundo. La retirada a la familia nuclear, a lo más privado es un fenómeno muy conocido en investigación sobre los parados. En estos meses nos parece que en algunos casos la parálisis empieza a curarse porque observamos que algunas personas recuperan antiguas amistades y algunos reanudan viejas o nuevas actividades sociales.

Pero la ruptura es mucho más extensa que estas pocas consideraciones sobre una parte del mundo de la vida que se deja entrever. Quisiera indicar breve- 
mente el carácter global de la ruptura tratando de la categoría confranza. La circunstancia que a partir de una cierta época, tal vez en los años sesenta, las condiciones sociales de la vida tenían, a pesar de toda la crítica del aprovisionamiento insuficiente con bienes de lujo, una estabilidad bastante grande, daba un gran vigor al mundo de la vida en la RDA. La gente no solamente tenía bastante de comer, sino que también algunos intereses sociales estaban relativamente bien satisfechos. Algunos ejemplos: los padres podían casi siempre confiar en la obtención de plazas para los niños en un jardín de infancia o en una guardería infantil durante las horas de trabajo. La central sindical FDGB procuraba plazas para las vacaciones en residencias o colonias en el mar o en las montañas. Era necesario esperar un largo tiempo (siete años, doce años) para obtener algunos bienes de alto consumo (congelador, coche, etc.), pero uno sabía que algún día le tocaría, sería su turno y recibiría el objeto deseado. Esta estabilidad ahora está totalmente cuestionada. Actualmente es difícil encontrar plaza en las guarderías infantiles. Uno puede realizar sus vacaciones en casi todas las partes del mundo y uno puede comprar todos los coches deseados si uno tiene dinero. Y esta no es la norma general excepto para pocos. Para la mayoría de la gente la seguridad característica del mundo de la vida hace falta ahora en esta uesfera de pertinencia» (según la interpretación de Schütz). En un sentido global la confianza característica para el mundo de la vida en el contexto social se ha resquebrajado.

Justamente en relación con la estabilidad relativa de la sociedad, se ha desarrollado una cierta confianza en el liderazgo comunista de la sociedad. Por esto muchas personas se vieron alentadas en la "idealización" del "continuar como siempren y permanecieron como bases de apoyo del régimen. Pero también el "continuar como siempre" indica un cierto tipo de confianza, Evidentemente el grado de confianza varía mucho. Fue relativamente alto en militantes sencillos, idealistas del partido que nunca habían nunca sentido las incidencias del estalinismo, $y$ este grado de confianza fue relativamente bajo entre bases de apoyo escépticos. En suma, hubo sin embargo en mucha gentc una confianza sólo relativa, que ahora después del derrumbamiento de la RDA y de la revelación de la verdadera situación, después del descubrimiento de las infamias del estalinismo y del conocimiento de que la estabilidad económica y social no podía perdurar mucho tiempo más - ahora esta confianza se convierte en una profunda decepción-. Justamente individuos que mantienen una fe en los ideales de una sociedad comunista se sienten profundamente engañados por el liderazgo del estado pretendidamente socialista. Muchos ocultan esta decepción lamentando lo perdido; la confrontación con la dureza cotidiana capiralista --determinada por la competición continua y el paropresenta las condiciones más estables de la RDA de color de rosa, pero a pesar de esto la decepción existe. Y se muestra, por ejemplo, en la dura crítica, muchas veces personalizante de los llamados "calcetines rojos", es decir, aquellos partidarios oportunistas del régimen que después de la derrota de la RDA han conseguido arrimar rápidamente el ascua a su sardina y pescar una buena colocación. 
La pérdida de confianza se reftere también a las relaciones sociales, porque, después de las revelaciones sobre el espionaje de la población por parte del servicio estatal de seguridad (Stasi) nadie actualmente sabe si un buen amigo o una buena amiga no fue al mismo tiempo un "IM": un colaborador informal, es decir, un/a espía del Stasi. No es raro que los individuos se retiren a la familia nuclear, a lo privado; pero también hay en algunos casos crisis de familia inducida por la ruptura del mundo de la vida.

A causa de la importación de condiciones sociales del oeste mucha gente se sicnte forastera en su propio país, lo que muestra de manera muy acusada el carácter global de la ruptura del mundo de la vida. Tal descripción tiene la desventaja de suscitar la impresión de que todos estuvieron igualmente afectados por los cambios descritos. Este no es el caso de ningún modo. Las diferencias son considerables: van desde parados sin ninguna seguridad ni estabilidad, a clientes ya más viejos de la asistencia pública, a empresarios, a nuevos ticos artibistas, que es como decir ganadores de la crisis. Una de las muchas diferencias que ha saltado especialmente a la vista en las interpretaciones de entrevistas, es la diferencia entre las generaciones. Para muchas personas mayores, adultas, es decir, la generación de los que tienen más de treinta y cinco años de edad, la ruptura del mundo de la vida tiene como consecuencia una crisis profunda de identidad. La desvalorización de cualificaciones profesionales, el paro y la crisis de las relaciones sociales han precipitado a mucha gente hacia una desesperación profunda. Están paralizados que es lo que sobrevenía. Mientras los más jóvenes, que no han vivido decenios de estabilidad relativa en la RDA, que han crecido en la era Gorbatchov con las esperanzas de un cambio positivo de los estados del este europeo pero también con las inseguridades que empezaban, estos jóvenes, que ponf́an muchas veces sus esperanzas en el cambio, no se han identificado con las condiciones existentes. Actualmente son mucho más flexibles y dispuestos a la adaptación e intentan con energía hallar su camino en las nuevas circunstancias sociales. Muchos elementos de la antigua sociedad de la RDA se habían hecho mundo de la vida sólo por la repetición y la habitualización. Para muchos jóvenes esta habitualización no fue bastante fuerte o simplemente no fue llevada a cabo, lo que hace que ahora sean más libres para la creación de nuevas conexiones, para un nuevo mundo de la vida. Pero para algunos la "liberación" es tan brutal que en vista del vacío de su mundo de la vida se evaden hacia grupos de extrema derecha, grupos de skinheads.

Las mujeres se hallan especialmente afectadas por el derrumbamiento de la confianza, porque para ellas en la RDA fue normal combinar trabajo profesional y trabajo de reproducción. Casi todas las mujeres tenían un puesto de trabajo en la RDA; ahora son las primeras en estar en paro, se ven reducidas al trabajo de casa (la famosa "vuelta al hornon) y por esto están limitadas y lesionadas de una manera doble: en la profesión y en el mundo de la vida.

Otra circunstancia ha contribuido a la destrucción del potencial de adaptación de la gente. En muchos alemanes del este existía a pesar de todo una voluntad fuerte de cambio y de nuevo comienzo. Estuvieron absolutamente 
dispuestos a aceptar una nueva situación social y nuevos elementos en su mundo de la vida, por un proceso de aprendizaje y de asimilación. Esta voluntad de cambio fue ahogada por el gobierno federal, por muchos alemanes del oeste que venian al este y especialmente por los Besser-Wessies. Fue ahogada con la introducción brutal y desde el punto de vista temporal, radical de las condiciones de la sociedad del oeste. Los Besser-Wessies no son solamente Wessies, es decir, gente del oeste sino que también lo saben todo mejor (besser) que los Ossies, la gente del este. La reunificación fue conducida con el gesto de "Aquí! Así es! No hay sutilezas!n, pero la decepción que ha provocado el cambio hace que la gente vuelva a la pasividad habitual.

Este fue uno de los elementos más importantes de la sociedad de la RDA: la pasividad de la gran mayoría provocada por la política, en principio paternalista, del viejo régimen. Este paternalismo iba de la tutela leve combinada con cuidados sociales, pasando por un control severo del estado policía, hasta la represión pura y dura según la sensibilidad del sector social en cuestión. Esta antigua impregnación del mundo de la vida se muestra todavía en muchos casos. Por ejemplo, hay parados que se dejan informar por las oficinas de colocación y se dejan rechazar en sus demandas. Si no están informados antes por los sindicatos o por otras personas, no tienen idea de que podrían oponerse y que podrían tener éxito, porque antes en casi ningún caso, una oposición tenía éxito. No están habituados al pensamiento de que pueden existir soluciones de compromiso, porque en la RDA en tales situaciones problemáticas la decisión fue ya - raramente - positiva, - en la mayoría de los casos- negativa, pero no de compromiso.

Muchos individuos que fueron activos en la sociedad de la RDA actualmente no lo son; no están preparados para continuar una actividad social porque ellos han vivido - como dicen en un resumen reflexivo- experiencias negativas con tales actividades. Hoy no tienen voluntad de comprometerse de nuevo porque su actividad antigua es difamada como soporte de un estado policía.

Ciertamente, existen también viejos vínculos que han perdurado tras la ruptura del mundo de la vida. Hay también elementos del mundo de la vida, ante todo en las familias, que son conservados; un agarrarse a viejas conexiones - por ejemplo a las fiestas de Navidad entre los antiguos colectivos de trabajo- porque ello muchas veces es necesario para sobrevivir. En suma y a pesar de todo se constata que la ruptura radical del mundo de la vida en Alemania del Este y probablemente de manera análoga en los otros países de la Europa del Este ha sacudido fuertemente a las personas y ha hecho caer a muchas en profundas crisis de identidad.

La fuerza de la convulsión en Alemania del Este por la ruptura del mundo de la vida es causada esencialmente por la velocidad del proceso de cambio. En general, los individuos no tenían un tiempo razonable, sensaro, para asimilar lo que vivian. Es cierto también que en un proceso de reconversión de larga duración el cambio del mundo de la vida sería radical y global, pero las posibilidades de asimilación podían haber sido mucho más grandes. 


\section{Ruptura y cambio}

La radicalidad de la ruptura del mundo de la vida en Alemania del Este y en otros países del este europeo no debería ocultar que también en Alemania del Oeste y en otros paises del oeste europeo existe un cambio del mundo de la vida. Este cambio lo califiqué hace unos años como mutación sociocultural, como pasaje del viejo modelo cultural a unos nuevo. Por esto quiero solamente indicar aquí que existen algunas publicaciones - de colegas y amigos, y publicaciones mías - que tratan de esta mutación. Hoy quiero solamente tomat en consideración algunas diferencias con la ruptura del mundo de la vida en Alemania del Este.

La mutación del mundo de la vida en Alemania del Oeste tiene otro carácter porqute es mucho más lenta. Además no es simultánea para todos los grupos de seres humanos, es decir que muchos, sobre todo la gente más adulta, viven todavía en el viejo modelo cultural mientras en otros sectores, ante todo entre los jóvenes, se vive un nuevo modelo cultural. El carácter de la mutación sociocultural en el oeste es también causado por el hecho que de manera univoca hay un cambio que viene del interior, una mutación inmanente mientras la colonialización de la Alemania del Este por el Oeste hace aparecer la ruptura como causada por el exterior. Por esto se octulta que en el último decenio de la RDA uno podía constatar fenómenos de erosión del interior, se oculta que la estabilidad del mundo de la vida estaba minada. Además hay que advertir que la ruptura al este hace aparecer la mutación en el oeste como menos grave.

Sin embargo es dificil ignorar que vivimos una crisis de la normalidad que de manera evidente concierne también al mundo de la vida. La crisis de la normalidad se puede definir en algunas palabras clave: es una crisis de la relación laboral normal, es decir, del contrato de trabajo fijo con ocho horas de trabajo o menos, de carácter ilimitado, etc. En lugar de esto tenemos más y más horarios laborables flexibles, trabajo a tiempo parcial, trabajo mediante subcontrato y contratos sin plazo señalado, en breve, relaciones laborales precarias. Valores antiguos como el trabajo pierden su función de dar sentido a la vida. El trabajo no lo es ya todo en la vida de las personas, el trabajo asalariado es objeto de un proceso de descentración. La crisis de normalidad es especialmente vistosa en la esfera de la familia: en lugar de la familia nuclear burguesa como modelo dominante se encuentra ahora una pluralización de estilos de vida que comprenden no sólo la antigua familia nuclear, sino también familias monoparentales y solteros o sueltos, y muchos otros. En muchas grandes ciudades alemanas el porcentaje de los solitarios en los hogares, alcanza ahora el $50 \%$ y más. La mutación de los vínculos sociales no concierne solamente a la familia, es una mutación global que se refiere también a la relación de individuos a partidos o sindicatos. Una palabra clave para esto es el aburrimiento de los partidos políticos que se hace sentir en la abstención o el fenómeno Ross Perot en los Estados Unidos, por ejemplo.

En las categorías del análisis def mundo de la vida se puede constatar que 
normas hasta ahora indudables llegan a ser problemáticas, que viejas capas de significación pierden su significación. A este cambio contribuyen factores exteriores como el paro, la reducción de los horarios de trabajo y muchos otros más, pero también factores subjetivos, porque el proceso de problematización no se para por sí mismo en alguna parte, sino que cuestiona también otras esferas de relevancia, y por tanto las hace problemáticas. La crisis de las viejas normas, el cuestionar las significaciones implicitas conduce a una gran exigencia, sobre todo de la generación más joven de comunicación, a una actitud comunicativa de fondo. Ahora todo debe legitimarse en la comunicación, sea la fe en Dios, sea la adhesión a un sindicato, sea simplemente el encuentro con la pandilla de amigos por la noche. La legitimación comunicativa del actuar llega a ser un proceso global y conduce a una mayor autorreflexividad de los seres humanos -y esto implica una esperanza.

Por cierto, la crisis de viejas normas y valores del viejo modelo cultural y la mutación del mundo de la vida, tanto en el este como en el oeste, lanza a los individuos a una libertad precaria y a la inseguridad. Muchos se sienten tan amenazados existencialmente por esta inseguridad que se evaden, en grupos de skinheads, en actos violentos de racismo. En otros, sin embargo, la inseguridad provoca una exigencia tan intensa de comunicación que puede conducir al descubrimiento del ideal de la comunicación sin dominación. La exigencia de relaciones sociales auténticas favorece también el esfuerzo de practicar la solidaridad en lo cotidiano.

La imagen de la mutación del mundo de la vida en Alemania, la imagen de esta sociedad en transición está compuesta por violencias de extrema derecha, pero también por las cadenas de luces** contra el racismo y el odio a los extranjeros, está compuesta por la búsqueda de chivos expiatorios por la inseguridad en el mundo de la vida por la que tantos individuos sufren pero también de muchos pequeños gestos de solidaridad cotidiana.

\section{Bibliografía}

Habermas, J. (1981). Theorie des kommunikativen Handelns, 2 Bde. Frankfurt/Main. SCHCTZ, A.; LuCKMANN, Th. (1979) Bd. 1, (1984) Bd. 2. Strukturen der Lebenswelt, Frankfurt/Main.

ZOL, R. (1992). Nouvel Individualisme et Solidarité Quotidienne, Paris: Ed. Kime.

** Manifestaciones en muchas ciudades alcmanas en la primavera de 1993, en protesta contra actos racistas. (Redacción) 